\title{
Observation of the two-channel quadrupolar Kondo effect in $\mathrm{Y}_{1-x} \mathrm{U}_{x} \mathrm{Pd}_{3}$
}

\author{
C. L. Seaman, M. B. Maple, B. W. Lee and S. Ghamaty \\ Department of Physics and Institute for Pure and Applied Physical Sciences, \\ University of California at San Diego, La Jolla, CA 92093 (USA)
}

M. S. Torikachvili

Department of Physics, San Diego State University, San Diego, CA 92182 (USA)

J.-S. Kang*, L. Z. Liu and J. W. Allen

Department of Physics, University of Michigan, Ann Arbor, MI 48109-1120 (USA)

D. L. Cox

Department of Physics, The Ohio State University, Columbus, OH 43210 (USA)

\begin{abstract}
We present measurements of electrical resistivity $\rho(T)$, magnetic susceptibility $\chi(T)$, and specific heat $C(T)$ for the system $\mathrm{Y}_{1-x} \mathrm{U}_{x} \mathrm{Pd}_{3}$. The data are consistent with nearly localized tetravalent uranium ions leading to "Fermi-level tuning" and competition between single ion Kondo $(0<x \leqslant 0.2)$ and cooperative spin glass-like $(0.3 \leqslant x \leqslant 0.5)$ behavior. The Kondo behavior is unusual and well described by the two-channel quadrupolar Kondo effect. At low temperatures $T \ll T_{\mathrm{K}}$, where $T_{\mathrm{K}}$ is the Kondo temperature, the electrical resistivity varies nearly linearly with temperature $\rho(T) / \rho(0) \approx 1-T /\left(a T_{\mathrm{K}}\right)$, and the electronic specific heat diverges logarithmically $\Delta C / T \approx-\left(1 / T_{\mathrm{K}}\right) \ln T$ with a finite residual $T=0$ entropy $S(0) \approx(R / 2) \ln 2$. This appears to be the first example of the quadrupolar Kondo effect and two-channel behavior in a dilute alloy and the first example of "marginal Fermi liquid" phenomenology in a fully three-dimensional system.
\end{abstract}

\section{Introduction}

The intermetallic compound $\mathrm{UPd}_{3}$ is unique in that it is the only material where all properties are consistent with localized $5 \mathrm{f}$ electrons. In particular, sharp crystalline electric field (CEF) levels are observed that are indicative of an $\mathbf{f}^{2}$ non-magnetic ground state and tetravalent uranium ions [1]. The linear coefficient of the low temperature specific heat $\gamma \approx 1 \mathrm{~mJ} \mathrm{~mol}^{-1} \mathrm{~K}^{-2}$ is typical of a normal metal [2]. Furthermore, photoemission spectroscopy (PES) and bremsstrahlung isochromat spectroscopy (BIS) measurements show a gap around the Fermi level $E_{F}$ with $5 f$ peaks below and above $E_{\mathrm{F}}$ [3]. In a recent $\mathrm{PES}$ study of $\mathrm{Y}_{1-x} \mathrm{U}_{x} \mathrm{Pd}_{3}$, the separation between $E_{\mathrm{F}}$ and the $5 \mathrm{f}$

*Present address: RIST, Pohang, 790-600, South Korea. 
peak $\left|E_{\mathrm{F}}-E_{6 \mathrm{f}}\right|$ was observed to decrease with decreasing $x$ as $\mathrm{UPd}_{3}$ was diluted with yttrium [4]. This was interpreted as "Fermi level tuning" in which substitution of trivalent yttrium for tetravalent uranium caused a decrease in the conduction electron density and therefore $E_{\mathrm{F}}$. Because the density of states at $E_{\mathrm{F}}, N\left(E_{\mathrm{F}}\right) \approx 1$ state $\mathrm{eV}^{-1}$ per cell, is low throughout the series [5], the drop in $E_{\mathrm{F}}$ is rather large (approximately $1 \mathrm{eV}$ as $x$ goes from 1 to 0 ). For moderately strong hybridization of the local $5 \mathrm{f}$ orbitals with the conduction band states such that a negative (antiferromagnetic) exchange interaction with an exchange constant $J \approx-\left\langle V_{\mathrm{kf}}{ }^{2}\right\rangle /\left|E_{\mathrm{F}}-E_{5 \mathrm{f}}\right|$ is generated, one might expect to observe an evolution from local moment behavior to heavy fermion or Kondo behavior. This expectation motivated the present study of the transport, magnetic and thermal properties of $\mathrm{Y}_{1-x} \mathrm{U}_{x} \mathrm{Pd}_{3}$. Indeed, our measurements are consistent with the Fermi-level tuning interpretation; the properties evolve smoothly toward Kondo behavior which is unusual and remarkably well described by a quadrupolar Kondo effect [6]. In addition, our most recent BIS data show that the Kondo behavior in the transport properties is accompanied by a growth of uranium $5 \mathrm{f}$ spectral weight just above $E_{\mathrm{F}}$, which we interpret as the associated Kondo resonance [7].

The quadrupolar Kondo effect was first developed by Cox in order to explain the anomalous magnetic properties of the cubic heavy fermion superconductor $\mathrm{UBe}_{13}$ [8]. In the simplest version of this microscopic model (which $\mathrm{Y}_{1-x} \mathrm{U}_{x} \mathrm{Pd}_{3}$ appears to satisfy), nearly localized, tetravalent uranium ions lie at sites of local cubic symmetry in which the CEF splits the Hund's rules ground ${ }^{3} \mathrm{H}_{4}$ multiplet into a non-magnetic $\Gamma_{3}$ doublet, $\Gamma_{4}$ and $\Gamma_{5}$ triplets, and a $\Gamma_{1}$ singlet, with $\Gamma_{3}$ lying lowest. The $\Gamma_{3}$ doublet admixes covalently with the conduction band states, leading to a Kondo effect; however, the antiferromagnetic exchange Hamiltonian is between the electric quadrupole moment (pseudospin-1/2) of the $\Gamma_{3}$ and two channels of time-reversed conduction band states. This Hamiltonian has the same form as the twochannel, spin 1/2 Kondo model and therefore the same thermodynamics except that the magnetic susceptibility is replaced by the quadrupolar susceptibility.

The two-channel $(m=2)$, spin $1 / 2(S=1 / 2)$ Kondo effect is a special case of the more general multichannel Kondo model in which a local impurity with spin $S$ couples to $m$ degenerate channels of conduction electrons [9]. The $m=2, S=1 / 2$ case is noteworthy in that it has the character of a local "marginal Fermi liquid" in which $\Delta C / T \approx-\ln (\alpha T)[10-12]$ and $\rho(T) /$ $\rho(0) \approx 1-A T$ [13], which has been postulated to explain the anomalous normal-state properties of certain heavy fermion and high- $T_{\mathrm{c}}$ superconductors $[14,15]$. In addition, $\Delta S(0)$ has the unusual value of $(R / 2) \ln 2[10-12]$. This work represents the first comprehensive set of data for any dilute alloy consistent with two-channel quadrupolar Kondo behavior, and the first observation of "marginal Fermi liquid" phenomenology [15] in a fully threedimensional system for which a candidate microscopic model exists. We hope that our work will stimulate further studies of this and related systems 
which may deepen our fundamental understanding of strongly correlated electronic materials.

\section{Experimental details}

Polycrystalline samples of $\mathrm{Y}_{1-x} \mathrm{U}_{x} \mathrm{Pd}_{3}$ were fabricated by arc melting the constituent elements in an ultrahigh purity argon atmosphere. X-ray diffraction patterns revealed no impurity peaks, except that samples with concentrations in the range $0.6 \leqslant x \leqslant 0.8$ exhibited peaks of both the hexagonal and cubic phases. Electrical resistivity $\rho(T)$ measurements were made using a standard four-wire, low frequency a.c. technique. Magnetoresistance measurements were made at various constant temperatures $0.3-15 \mathrm{~K}$ in fields up to $6 \mathrm{~T}$ at UCSD $(x=0.1$ and 0.2$)$ and up to $30 \mathrm{~T}$ at the Francis Bitter National Magnet Laboratory at MIT $(x=0.2)$. Magnetic susceptibility $\chi(T)$ and magnetization measurements in fields up to $6 \mathrm{~T}$ were performed with both a commercial SQUID and a Faraday magnetometer. Specific heat $C(T)$ was measured in a semi-adiabatic heat pulse calorimeter in the temperature range $0.5 \leqslant T \leqslant 30 \mathrm{~K}$.

\section{Phase diagram}

Figure 1 shows the temperature vs. uranium concentration $(T-x)$ phase diagram of $\mathrm{Y}_{1-x} \mathrm{U}_{x} \mathrm{Pd}_{3}$ which summarizes the general behavior as a function of $x$. For $0.9 \leqslant x \leqslant 1.0, \mathrm{Y}_{1-x} \mathrm{U}_{x} \mathrm{Pd}_{3}$ forms the hexagonal $\mathrm{Ni}_{3} \mathrm{Ti}$ crystal structure in which the uranium ions lie at two inequivalent sites of local hexagonal and quasi-cubic symmetry, while for $0 \leqslant x \leqslant 0.5$, it forms the cubic $\mathrm{Cu}_{3} \mathrm{Au}$ structure in which there is only one uranium site of local cubic symmetry. There is a mixed phase region for $0.6 \leqslant x \leqslant 0.8$ in which single-phase samples could not be fabricated. The cubic lattice parameter $a$ increases by less than $1 \%$ (from approximately 4.06 to $4.09 \AA$ ) as $x$ increases from 0 to 0.5 . Such a small increase in consistent with uranium ions that are tetravalent, being comparable in size with $\mathrm{Y}^{3+}$ ions and much smaller than trivalent uranium ions. We remark that $\mathrm{UPd}_{4}$ forms the same $\mathrm{Cu}_{3} \mathrm{Au}$ structure except that palladium ions occupy some of the uranium sites, forming $\mathrm{Pd}_{0.2} \mathrm{U}_{0.8} \mathrm{Pd}_{3}$ [16]. The compound $\mathrm{UPd}_{4}$ might therefore be expected to have similar properties to $\mathrm{Y}_{0.2} \mathrm{U}_{0.8} \mathrm{Pd}_{3}$, if it could be fabricated with the cubic structure.

The hexagonal samples $(x=0.9$ and 1.0) appear to have non-magnetic ground states, although $\mathrm{UPd}_{3}$ exhibits some type of sample-dependent, firstorder transition at $T \approx 7 \mathrm{~K}$ which has been speculated to be structural, possibly involving quadrupolar ordering $[2,17,18]$. The cubic samples with higher uranium concentrations exhibit some type of spin- (or possibly quadrupolar-) glass freezing. The magnetic susceptibility $\chi(T)$ is irreversible below a temperature $T_{\mathrm{SG}}=4.5 \mathrm{~K}, 10 \mathrm{~K}$ and $21 \mathrm{~K}$ for $x=0.3,0.4$ and 0.5 respectively, denoted in Fig. 1. Both the irreversible $\chi(T)$ and the specific heat data $C(T)$ 


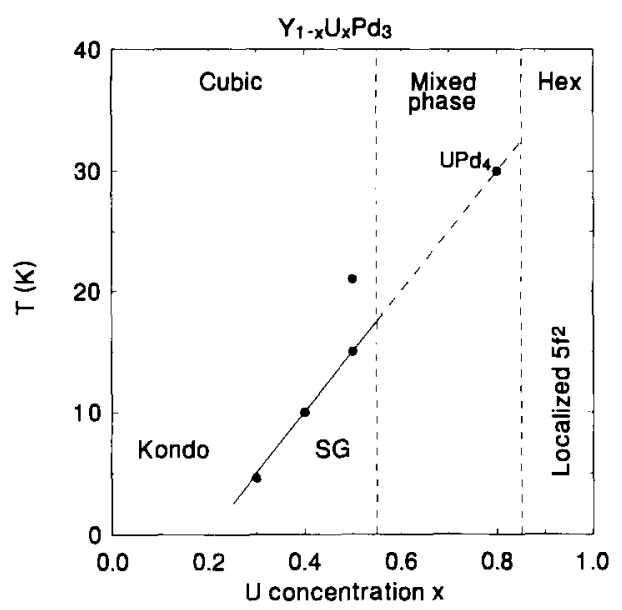

Fig. 1. Phase diagram for $\mathrm{Y}_{1-x} \mathrm{U}_{x} \mathrm{Pd}_{3}$. Samples form the cubic $\mathrm{Cu}_{3} \mathrm{Au}$ crystal structure for $0 \leqslant x \leqslant 0.5$ and the hexagonal $\mathrm{Ni}_{3} \mathrm{Ti}$ structure for $0.9 \leqslant x \leqslant 1$. The Kondo behavior for $x \leqslant 0.2$ is well described by the quadrupolar Kondo effect, characterized by the Kondo temperature $T_{\mathrm{K}}$ which decreases with $x$ owing to Fermi-level tuning. At higher concentrations, cubic samples exhibit spin glass-like cooperative behavior below the temperature $T_{\mathrm{SG}}$, defined from the irreversible $\chi(T) . T_{\mathrm{SG}}(x)$ extrapolates to the Néel temperature of $\mathrm{UPd}_{4}$. The hexagonal samples are consistent with localized $5 f^{2}$ uranium ions with a non-magnetic ground state.

for $x=0.5$ form a double-peak structure, possibly owing to sample inhomogeneity, and are both indicated in Fig. 1. The monotonic increase with $x$ is expected for RKKY interactions whose strength increases as approximately $J^{2} N\left(E_{\mathrm{F}}\right) x$. For $x=0.2, \chi(T)$ is reversible down to at least $0.4 \mathrm{~K}$, the low temperature limit of the Faraday magnetometer. Also shown in Fig. 1 is an extrapolation through the $T_{\mathrm{SG}}(x)$ points which passes near the Néel temperature of $\mathrm{UPd}_{4}$ and strengthens our suggestion that $\mathrm{UPd}_{4}$ might be considered as part of this series of compounds. The ordered moment of $\mathrm{UPd}_{4}$ has been determined to be $g J \mu_{\mathrm{B}}=0.8 \mu_{\mathrm{B}}$ from neutron diffraction studies [16]. Evidence has been given for localized CEF states in $\mathrm{UPd}_{4}$ by means of inelastic neutron scattering (INS) [19] with an excited state $\Gamma_{5}$ triplet lying approximately 5.1 $\mathrm{meV}(60 \mathrm{~K})$ above the $\Gamma_{3}$ ground state doublet.

For $0<x \leqslant 0.2, \mathrm{Y}_{1-x} \mathrm{U}_{x} \mathrm{Pd}_{3}$ exhibits unusual Kondo behavior which can be explained very well by a two-channel, spin $1 / 2$ Kondo effect, characterized by a Kondo temperature $T_{\mathrm{K}}$ that increases with decreasing $x$. This is consistent with the Fermi-level interpretation of the PES data since the nearly linear decrease in the binding energy with decreasing $x$ should cause a large increase in $T_{\mathrm{K}}$ since $T_{\mathrm{K}} \approx E_{\mathrm{F}} \exp \left[-1 /|J| N\left(E_{\mathrm{F}}\right)\right] \approx E_{\mathrm{F}} \exp \left[-\left|E_{\mathrm{F}}-E_{5 \mathrm{f}}\right| /<V_{\mathrm{kf}}^{2}>N\left(E_{\mathrm{F}}\right)\right]$. It is also consistent with the Kondo-resonance interpretation of our BIS data [7], since the intensity of the new peak above $E_{F}$ increases with decreasing $x$. Rigorous group theoretical calculations show that any two-channel, spin $1 / 2$ Kondo effect must be quadrupolar for uranium ions that are tetravalent with local cubic or tetragonal symmetry. We now describe in more detail 
our results of $\rho(T), \chi(T)$ and $C(T)$ measurements, concentrating on data for $x \leqslant 0.2$ to show how they manifest the quadrupolar Kondo effect.

\section{Electrical resistivity}

Shown in Fig. 2 is the temperature dependence of the normalized resistance of $\mathrm{Y}_{1-x} \mathrm{U}_{x} \mathrm{Pd}_{3}$ for temperatures $1.2 \leqslant T \leqslant 300 \mathrm{~K}$. Values of the room temperature resistivities for samples with various $x$ are estimated, within $5 \%$, as follows:
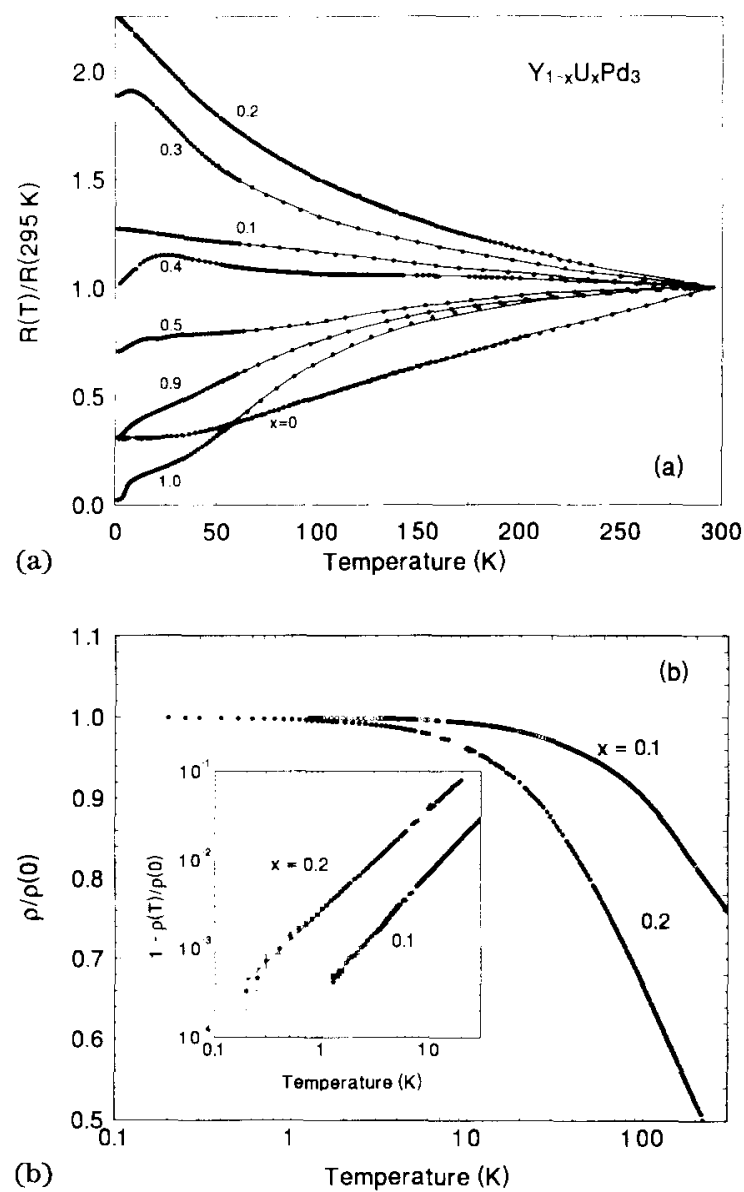

Fig. 2. (a) Temperature dependence of the normalized electrical resistivity. Despite the mixed phase region, $R(T)$ evolves smoothly as a function of $x$. The onset of glass freezing causes the resistance to decrease at low temperature for $x=0.3,0.4$ and 0.5 . (b) Normalized electrical resistivity $\rho / \rho(0)$ vs $\ln T$, indicative of the Kondo effect. Inset: the data follow power law behavior at low temperatures $(T<20 \mathrm{~K}): \rho(\mathrm{T}) / \rho(0)=1-\left(T / T_{0}\right)^{\mathrm{n}}$ where for $x=0.2\{x=0.1\}$, $n=1.13 \pm 0.04\{1.30 \pm 0.05\}$ and $T_{0}=180 \pm 20 \mathrm{~K}=(4.3 \pm 0.5) T_{\mathrm{K}}\left\{400 \pm 90 \mathrm{~K}=(1.8 \pm 0.4) T_{\mathrm{K}}\right\}$, where $T_{\mathrm{K}}=42 \mathrm{~K}\{220 \mathrm{~K}\}$ (see Fig. 5). For a single-channel, $S=1 / 2$ magnetic Kondo effect, one would expect $T^{2}(n=2)$ saturation at the lowest temperatures; while for the two-channel Kondo effect, theory predicts a linear saturation $(n=1)$ and $T_{0}=4.7 T_{\mathrm{K}}$. 
$(x, \rho(\mu \Omega \mathrm{cm}))=(0,13),(0.1,50),(0.2,136),(0.3,98),(0.4,92),(0.5,68)$, $(0.9,96),(1.0,130)$. Whereas $\mathrm{YPd}_{3}$ behaves as a normal metal, the samples with $x=0.1$ and 0.2 have a negative temperature coefficient, suggestive of a Kondo effect. A sample with $x=0.02$ (not shown) also had a negative temperature coefficient below the Kondo minimum at $T \approx 200 \mathrm{~K}$. The $x=0.3$ sample behaves similarly above $20 \mathrm{~K}$, but develops a peak at lower temperatures owing to the onset of cooperative behavior reminiscent of spin-glass freezing. This peak occurs at successively higher temperatures for $x=0.4$ and 0.5 . Concurrently, the Kondo behavior diminishes, evolving smoothly as $x$ increases toward the behavior of $\mathrm{UPd}_{3}$ which, like $\mathrm{YPd}_{3}$, is that of a normal metal. The drop in $\rho$ below approximately $7 \mathrm{~K}$ of $\mathrm{UPd}_{3}$ coincides with the firstorder transition and has been observed before [2]. The similarity between the cubic $x=0.5$ and the hexagonal $x=0.9$ samples is striking, suggesting that the Fermi level tuning controls the evolution of the low temperature properties. The evolution of $\rho(T) v s . x$ reflects the competition between the Kondo (single impurity limit) and RKKY (interionic) interactions for the ground state: $T_{\mathrm{K}}$ decreases with $x$ while $T_{\mathrm{SG}}$ increases with $x$; cooperative behavior wins out for $x \geqslant 0.3$. This type of evolution in $\rho(T) v s . x$ has been seen in other systems, e.g. $\mathrm{La}_{1-x} \mathrm{Ce}_{x} \mathrm{~B}_{6}$ [20], and is well described by theoretical calculations in terms of this type of competition [21].

An unusual aspect of the glass freezing is the occurrence of a sharp anomaly in $\rho(T)$ precisely at $T_{\mathrm{SG}}$ determined from the irreversibility in $\chi(T)$. Such an anomaly is not usually seen in spin glass systems. A possible explanation is the simultaneous occurrence of a structural transition, such as a Jahn-Teller distortion, which might be electronically driven by the stable $\Gamma_{3}$ ground state doublet $[8,11,22]$. We find the magnetic nature of the glassy state puzzling and in need of further characterization. Something similar could be happening in $\mathrm{UPd}_{3}$ below around $7 \mathrm{~K}$.

A plot of $\rho$ vs. $\ln T$ in Fig. 2(b) demonstrates the Kondo-like behavior for $x=0.1$ and 0.2 . The background contribution has not been subtracted, but is expected to be rather small as estimated from the resistivity of $\mathrm{YPd}_{3}$ which has a value of approximately $13 \mu \Omega \mathrm{cm}$ at $300 \mathrm{~K}$ and decreases with decreasing temperature, saturating to a constant value around $4 \mu \Omega \mathrm{cm}$ below approximately $25 \mathrm{~K}$. For the samples with $x=0.1$ and 0.2 , as $T$ decreases there is a logarithmic increase in $\rho$ which begins to saturate below $T \approx T_{\mathrm{K}}$. This logarithmic increase is expected to occur near $T \approx T_{\mathrm{K}}$ for both the $m=1$ and $m=2$ models, although at such high temperatures CEF effects make it difficult to estimate $T_{\mathrm{K}}$ precisely. For the usual single-channel $m=1$, $S=1 / 2$ magnetic Kondo effect, $T_{\mathrm{K}}$ is given as the midpoint of this rise, yielding an estimate of $T_{\mathrm{K}} \approx 200 \mathrm{~K}$, and at lowest temperatures $\left(T \leq 0.1 T_{\mathrm{K}} \approx 20\right.$ $\mathrm{K}), \rho(T) / \rho(0) \approx\left[1-\left(T / T_{0}\right)^{n}\right]$ saturates quadratically where $n=2$. In contrast, a heuristic argument for the $m=2, S=1 / 2$ model leads to a low temperature resistivity of the form $\left.\rho(T) / \rho(0) \approx\left[1-(1+\ln 2) T / 8 T_{\mathrm{K}}\right)\right]$, where $n=1$ and $T_{0}=4.7 T_{\mathrm{K}}$ [13]. In the inset of Fig. $2(\mathrm{~b})$, we show a $\ln (1-\rho / \rho(0)) v s . \ln T$ plot of the data for $x=0.2\{x=0.1\}$ from $0.2 \mathrm{~K}\{1.2 \mathrm{~K}\}$ to $20 \mathrm{~K}$ in which we find $n=1.13 \pm 0.04\{1.30 \pm 0.05\}$ and $T_{0}=180 \pm 20 \mathrm{~K}=(4.3 \pm 0.5) T_{\mathrm{K}}$ 
$\left\{400 \pm 90 \mathrm{~K}=(1.8 \pm 0.4) T_{\mathrm{K}}\right\}$, where $T_{\mathrm{K}}=42 \mathrm{~K}\{220 \mathrm{~K}\}$ was determined from the specific heat data presented below. The uncertainties in $n$ and $T_{0}$ have been estimated from the uncertainty in $\rho(0)$, which was chosen to give the best straight lines shown in the figure. The saturation of the data is seen to be much closer to being linear than quadratic. For $x=0.02$, the rise in $\rho(T)$ with decreasing $T$ is also approximately linear down to $1.2 \mathrm{~K}$, but the experimental resolution was not sufficient to determine the power law behavior more precisely. The value of $T_{0}$ for the more linear sample, $x=0.2$, is also consistent with the two-channel formula. For the $x=0.1$ sample, the larger value of $T_{0}$ is consistent with a larger $T_{\mathrm{K}}$ value than for $x=0.2$. The deviation from the two-channel formula may be due to this larger $T_{\mathrm{K}}$ value which should be of the same order as the CEF excited states. Still, the saturation is less than quadratic down to temperatures $T \approx 0.005 T_{\mathrm{K}}$.

The magnetoresistance $\Delta R(H) / R(0)=\rho(H) / \rho(0)-1$ of the $x=0.2$ specimen is negative and small, in agreement with other recent measurements [23], and is well described by the equation $\Delta R(H) / R(0)=-\left(H / H_{0}\right)^{2}$ for $H \leqslant 6$ $\mathrm{T}, 0.3 \mathrm{~K} \leqslant T \leqslant 15 \mathrm{~K}$. The coefficient $H_{0}$ increases monotonically with temperature from approximately $80 \mathrm{~T}$ at $T=0.3 \mathrm{~K}$ to approximately $140 \mathrm{~T}$ at $T=15 \mathrm{~K}$. At $T=0.6 \mathrm{~K}$ and $H=30 \mathrm{~T},|\Delta R(30 \mathrm{~T}) / R(0)| \lesssim 5 \%$. The magnetoresistance of the $x=0.1$ sample is zero within the experimental resolution for $H \leqslant 6 \mathrm{~T}$, $0.3 \mathrm{~K} \leqslant T \leqslant 15 \mathrm{~K}$; at $T=0.3 \mathrm{~K}$ this corresponds to $|\Delta R(6 \mathrm{~T}) / R(0)|<0.03 \%$. It is presently unclear exactly what to expect theoretically for the quadrupolar Kondo effect; however, the negligible magnetoresistance of the $x=0.1$ sample seems consistent with having a larger $T_{\mathrm{K}}$ value than the $x=0.2$ sample. Moreover, any magnetic Kondo effect would yield a strong negative magnetoresistance.

\section{Magnetic susceptibility}

Figure 3 shows a plot of the temperature dependence of the inverse magnetic susceptibility $\chi^{-1}$ below $300 \mathrm{~K}$. The data were fitted to a Curie-Weiss law plus a constant from $100 \mathrm{~K}$ to $300 \mathrm{~K}: \chi=\chi_{0}+C /\left(T-\theta_{\mathrm{CW}}\right)$. The effective moment $\mu_{\text {eff }}$ and $\chi_{0}$ were approximately constant across the series with mean values $\mu_{\mathrm{eff}}=3.4 \pm 0.1 \mu_{\mathrm{B}}$ and $\chi_{0}=-6 \pm 1 \times 10^{-4} \mathrm{emu}(\mathrm{mol} \mathrm{U})^{-1}$. The compound $\mathrm{YPd}_{3}$ is diamagnetic with a value $\chi_{0}=-6 \times 10^{-5} \mathrm{emu} \mathrm{mol}{ }^{-1}$, in agreement with previous measurements [24]. The most enlightening finding is the evolution of the Curie-Weiss temperature $\theta_{\mathrm{CW}}$, which is negative, indicating antiferromagnetic correlations, with a magnitude that decreases rapidly with $x$ from approximately $430 \mathrm{~K}(x=0.1)$ to approximately $150 \mathrm{~K}(x=0.2)$ to approximately $60 \mathrm{~K}(x=1.0)$. This evolution is responsible for the downward shift in $\chi^{-1}(T)$ with $x$ in Fig. 3. If $\theta_{\mathrm{CW}}$ reflected interionic AFM correlations, one would expect it to increase in magnitude with $x$. We therefore believe $\theta_{\mathrm{CW}}$ reflects $T_{\mathrm{K}}$; for Kondo systems, typically $\left|\theta_{\mathrm{CW}}\right| \approx(3-4) T_{\mathrm{K}}$. This trend agrees with the Fermi-level tuning aspect of this system.

At low temperatures, $\chi(T)$ is irreversible below $4.5 \mathrm{~K}, 10 \mathrm{~K}$ and $21 \mathrm{~K}$ for $x=0.3,0.4$ and 0.5 respectively, as measured in a field of 100 Oe. For 


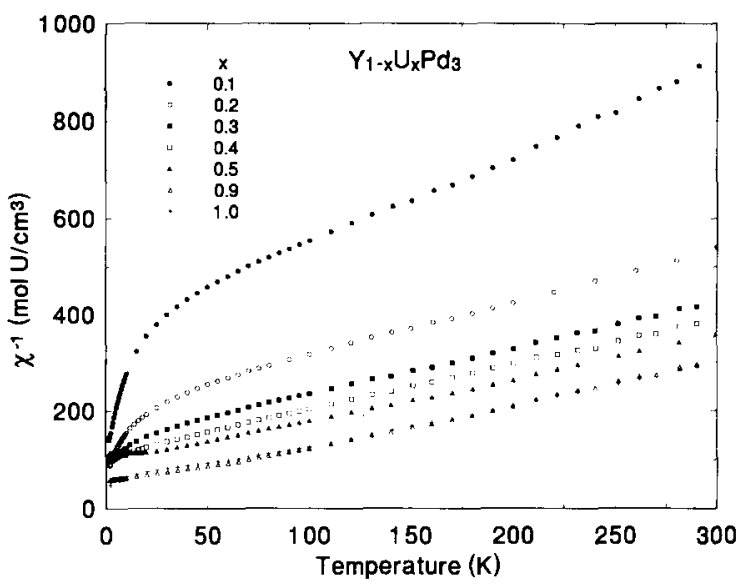

Fig. 3. Inverse magnetic susceptibility per mol of uranium $v$ s temperature. At high temperatures $100 \mathrm{~K} \leqslant T \leqslant 300 \mathrm{~K}$, the data follow a Curie-Weiss law plus a constant: $\chi=\chi_{0}+C /\left(T-\theta_{\mathrm{CW}}\right)$, where $\mu_{\mathrm{eff}}=3.4 \pm 0.1 \mu_{\mathrm{B}}$ and diamagnetic constant $\chi_{0}$ are approximately independent of $x$. The Curie-Weiss temperature $\theta_{\mathrm{CW}}$ is negative, indicative of antiferromagnetic correlations, with a magnitude that decreases monotonically with $x$. This is consistent with Fermi-level tuning and a Kondo effect in which $T_{\mathrm{K}}$ decreases monotonically with $x$.

$x=0.2, \chi(T)$ was found to be reversible down to at least $0.4 \mathrm{~K}$, the low temperature limit of the Faraday magnetometer. For this sample, $\chi$ continues to increase as $T$ decreases, quite unlike the usual $m=1, S=1 / 2$ Kondo effect in which the local moments are screened by the conduction electrons, forming a local Fermi liquid, many-body singlet ground state, and causing $\chi(T)$ to saturate to a constant value at low temperatures $T \ll T_{\mathrm{K}}$. For the quadrupolar Kondo effect, large van Vleck contributions to the susceptibility are believed to give $\Delta \chi \approx A-B T^{1 / 2}$ [13]. While our data follow $T^{1 / 2}$ behavior between 1.6 $\mathrm{K}$ and $4 \mathrm{~K}$, they rise somewhat more steeply than $-\ln T$ below $T=1.0 \mathrm{~K}$. A linear fit of $\chi^{-1}(T)$ for $0.8 \mathrm{~K} \leqslant T \leqslant 1.6 \mathrm{~K}$ yielded $\mu_{\text {eff }} \approx 0.6 \mu_{\mathrm{B}}$, which is comparable with that of the $\Gamma_{4}$ triplet $\left(\mu_{\mathrm{eff}}=0.69 \mu_{\mathrm{B}}\right)$, and $\theta_{\mathrm{CW}} \approx-1.0 \mathrm{~K}$. However, it is possible that paramagnetic impurities contribute significantly to $\chi(T)$ at low temperatures, obscuring the intrinsic temperature dependence of $\chi$ for $\mathrm{Y}_{0.8} \mathrm{U}_{0.2} \mathrm{Pd}_{3}$. Indeed, such a contribution is seen in the pure $\mathrm{YPd}_{3}$ sample.

\section{Specific heat}

Figure 4 shows electronic specific heat data for $\mathrm{Y}_{1-x} \mathrm{U}_{x} \mathrm{Pd}_{3}$ plotted as $\Delta C / T$ vs $T$. The electronic specific heat $\Delta C$ was obtained by subtracting an estimated phonon background term $\beta T^{3}$, with $\beta$ obtained by scaling the value of $\mathrm{YPd}_{3}\left(\beta_{0}=0.256 \mathrm{~mJ} \mathrm{~mol}^{-1} \mathrm{~K}^{-4}\right)$ [25] by the average formula unit molecular weight for a given $x$. Extrapolation of this result to $x=1$ gives $\beta=0.41 \mathrm{~mJ}$ $\mathrm{mol}^{-1} \mathrm{~K}^{-4}$, to be compared with isostructural (to $\mathrm{UPd}_{3}$ ) $\mathrm{ThPd}_{3}$ which has $\beta=0.5 \mathrm{~mJ} \mathrm{~mol}^{-1} \mathrm{~K}^{-4}$ [2]. The phonon contribution could not be determined 


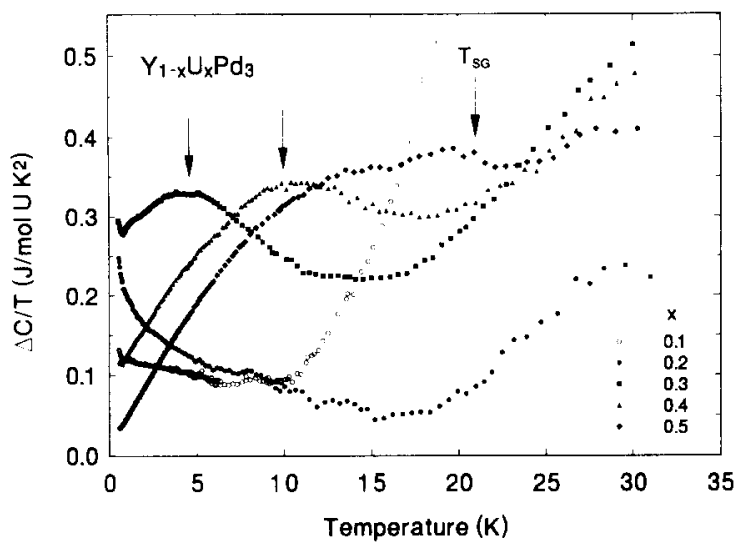

Fig. 4. Temperature dependence of the electronic specific heat per mole of uranium, $\Delta C / T$ vs. $T$. The temperature $T_{\mathrm{SG}}$ is the peak position of $\Delta C(T) / T$ associated with apparent spinglass freezing; $\chi(T)$ shows an onset to irreversibility at the same temperature. The upturn near $20 \mathrm{~K}$ is apparently due to an excited state Schottky anomaly. The entropies associated with these peaks converge to approximately $R \ln 2$ near $T \approx 20 \mathrm{~K}$, suggestive of freezing out of a doublet ground state. For $x=0.1$ and 0.2 , there is no peak and the entropy appears to saturate to $(R / 2) \ln 2$ near $T \approx 20 \mathrm{~K}$, suggestive of a ground state entropy of the same value.

directly owing to the apparent Schottky anomaly above around $20 \mathrm{~K}$. However, the low temperature data are insensitive to $\beta$. For $x=0.3,0.4$ and 0.5 , a broad peak in $\Delta C / T$ occurs at $T_{\mathrm{SG}}$, as is usual for spin-glass systems. The entropies under these curves converge to $R \ln 2$ at $20 \mathrm{~K}$ and fall nearly on top of each other at higher temperatures, suggesting that the spin-glass freezing removes entropy from a doublet ground state. For $\mathrm{U}^{4+}$ with local cubic symmetry, this implies that the $\Gamma_{3}$ doublet lies lowest. We ascribe the increase in $\Delta C / T$ at higher temperatures to a Schottky anomaly. The data for $x=0.1$ and 0.2 are qualitatively quite different from those of the spinglass samples. There is no peak in $\Delta C / T$ down to $0.5 \mathrm{~K}$, the lowest temperature attained; $\Delta C / T$ does not even saturate at low temperatures, as expected for the $m=1, S=1 / 2$ Kondo effect, but continues to diverge logarithmically. As shown in Fig. 5, this logarithmic divergence occurs over more than a decade of temperature. Furthermore, the $\Delta C / T$ data are greatly reduced for the $x=0.1$ and 0.2 samples; the entropy approaches $(R / 2) \ln 2$ above $T=20 \mathrm{~K}$ [6]. A similar Schottky anomaly is observed at higher temperatures, although the data for the $x=0.1$ sample seem to deviate from the behavior of the other samples. We believe this is an artifact of incorrectly subtracting the large background phonon contribution of $\mathrm{YPd}_{3}$, which becomes more important for samples with lower uranium concentration $x$, and also at higher temperatures. However, we emphasize that the temperature dependence of $\Delta C / T$ below around $10 \mathrm{~K}$ should not be sensitive to this subtraction. This specific heat result is the most significant piece of evidence we have for two-channel behavior in $\mathrm{Y}_{1-x} \mathrm{U}_{x} \mathbf{P d}_{3}$. 


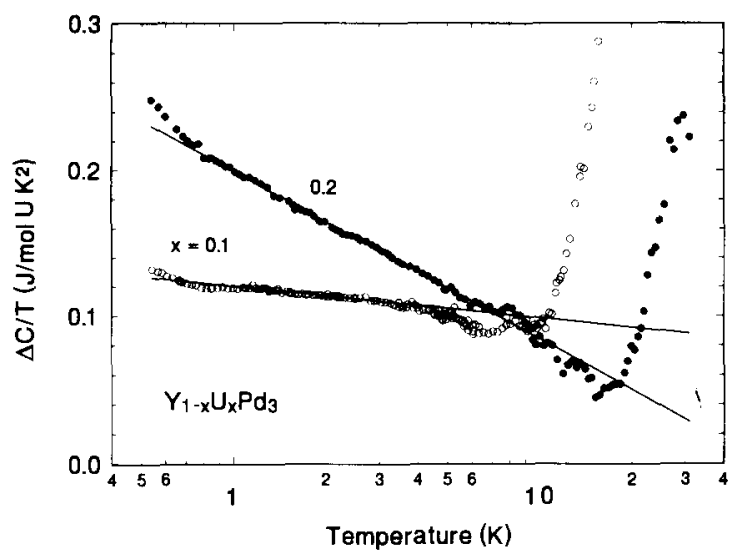

Fig. 5. Electronic specific heat $\Delta C / T$ vs. $\ln T$ for $x=0.1$ and 0.2 . The solid lines represent linear least squares fits of the data to the form $\Delta C / T=-\left(0.25 / T_{\mathrm{K}}\right) \ln \left(T / 0.41 T_{\mathrm{K}}\right)+b[11]$. From the slopes we obtain $T_{\mathrm{K}}=220 \mathrm{~K}$ and $42 \mathrm{~K}$ respectively. The background contribution $b \approx 100$ $\mathrm{mJ} \mathrm{mol} \mathrm{mol}^{-1} \mathrm{~K}^{-2}$ probably arises from an excited state Schottky anomaly.

Such a logarithmic divergence in $\Delta C / T$ has been predicted theoretically to occur for the $m=2, S=1 / 2$ Kondo effect, which can be manifested through the quadrupolar Kondo effect. We have fit our data to the theoretical model plus a background term $b: \Delta C(T) / T=-\left(0.251 / T_{\mathrm{K}}\right) \ln \left(T / 0.41 T_{\mathrm{K}}\right)+b$. For $x=0.2$ $(x=0.1)$ we find $T_{\mathrm{K}}=42 \mathrm{~K}(220 \mathrm{~K})$ and $b=61(115) \mathrm{mJ}(\mathrm{mol} \mathrm{U})^{-1} \mathrm{~K}^{-2}$. These values for $T_{\mathrm{K}}$ are comparable with what one might estimate from the resistivity data and we note that the larger value of $T_{\mathrm{K}}$ for $x=0.1$ is consistent with Fermi-level tuning. The background term could be due to the overlapping Schottky anomaly which could result from a $\Gamma_{5}$ triplet approximately $60 \mathrm{~K}$ above the ground $\Gamma_{3}$ doublet. A $\Gamma_{5}$ triplet at around $60 \mathrm{~K}$ would give a value of $\chi(0)$ comparable with the measured value. Furthermore, the two-channel model has a finite residual $T=0$ entropy $S(0)=(R / 2) \ln 2$, which is in remarkable agreement with our data for $x=0.1$ and 0.2 .

Recent new results also support a quadrupolar Kondo effect in $\mathrm{Y}_{1-x} \mathrm{U}_{x} \mathrm{Pd}_{3}$. Magnetic specific heat data show a crossover characteristic of coupling to channel spin [23]. Furthermore, preliminary magnetic neutron scattering data show no pronounced quasielastic scattering intensity but do show CEF peaks consistent with a $\Gamma_{5}$ triplet at approximately $5 \mathrm{meV}$ and a $\Gamma_{4}$ triplet at approximately $24 \mathrm{meV}$ [26]. While the $\chi(T)$ and $\rho(H, T)$ results await complete understanding, it is clear that a magnetic Kondo effect would produce a spin dependent crossover of the specific heat in field and a quasielastic magnetic scattering, in contrast to the data.

Experiments are in progress to test further our interpretation of the quadrupolar Kondo effect and characterize further this remarkable system, including more complete INS, ultrasonic attenuation measurements to determine the temperature dependence of the quadrupolar susceptibility, nuclear magnetic resonance, a muon spin relaxation investigation of the glassy state, and investigation of similar alloys such as $\mathrm{Y}_{1-x} \operatorname{Pr}_{x} \mathrm{Pd}_{3}$ and $\mathrm{Y}_{1-x}(\mathrm{U}, \mathrm{Th})_{x} \mathrm{Pd}_{3}$. 
Finally, we remark on two related systems. The first observation of the Kondo effect in a dilute actinide alloy was in the cubic system (La, U) $\mathrm{Al}_{2}$ with less than $500 \mathrm{ppm}$ uranium solubility and $T_{\mathrm{K}} \approx 10 \mathrm{~K}$ [27]. For tetravalent uranium ions, this could be a quadrupolar Kondo effect. More recently, it was proposed that the itinerant 5 f-electron antiferromagnetic $\mathrm{NpSn}_{3}$, which forms the $\mathrm{Cu}_{3} \mathrm{Au}$ cubic crystal structure, should be viewed as a concentrated Kondo system, similar to $\mathrm{CeAl}_{2}$ [28]. The argument was that the ordered moment of $0.3 \mu_{\mathrm{B}}$ for a localized $\mathrm{Np}^{3+}\left(5 \mathrm{f}^{4}\right)$ non-Kramer's ion with a ${ }^{5} \mathrm{H}_{4}$ Hund's rules ground state that is split by the cubic CEF is too small compared with the calculated values for either the $\Gamma_{4}$ or $\Gamma_{5}$ magnetic triplets. Again, any Kondo effect by a $J=4$ ion with local cubic symmetry could be quadrupolar.

\section{Conclusion}

In summary, $\mathrm{Y}_{1-x} \mathrm{U}_{x} \mathrm{Pd}_{3}$ appears to be the first example of a dilute alloy to exhibit the two-channel quadrupolar Kondo effect, as particularly exemplified by $x=0.2$. This results from nearly localized tetravalent uranium ions with CEF-split $5 f$ levels that are covalently hybridized with the conduction band states, generating an antiferromagnetic exchange interaction which leads to the Kondo effect. We believe $\Gamma_{3}$ lies lowest. The electrical resistivity data leave little doubt that a Kondo effect occurs; yet the data cannot be well described by the usual single-channel spin-1/2 magnetic Kondo model. The data are remarkably well described by a two-channel, spin-1/2 Kondo model. For $\mathrm{U}^{4+}$ ions with local cubic symmetry which exhibit a two-channel, spin$1 / 2$ Kondo effect, the only such possibility is provided by a quadrupolar Kondo effect. The magnetoresistance, field dependent specific heat data [23], and INS results appear to exclude the possibility that the two-channel behavior results from a magnetic two-channel Kondo effect.

\section{Acknowledgments}

We acknowledge enlightening discussion with I. Affleck, Y. Dalichaouch, A. Ludwig, D. E. MacLaughlin, H. A. Mook, G. Nieva and P. Schlottmann. We thank K. Flynn for technical assistance. This research was supported by the US National Science Foundation under Grant Nos. DMR-87-21455 (UCSD) and DMR-87-21654 (UM), the US Department of Energy under Grant Nos. DE-FG03-86ER45230 (UCSD) and DE-FG02-87ER45326 (OSU), and an A. P. Sloan Foundation Research Fellowship (OSU). One of us (SG) gratefully acknowledges partial support from UNOCAI Corporation.

\section{References}

1 N. Shamir, M. Melamud, H. Shaked and M. Weger, Physica B, 94 (1978) 225. A. F. Murray and W. J. L. Buyers, in J. E. Crow, R. P. Guertin and T. W. Mihalisin (eds.), Crystalline Electric Field and Structural Effects in f-Electron Systems, Vol. 1, Plenum, New York, 1979, p. 257. 
W. J. L. Buyers, A. F. Murray, T. M. Holden, E. C. Svensson, P. de V. Duplessis, G. H. Lander and O. Vogt, Physica B, 102 (1980) 291.

W. J. L. Buyers, T. M. Holden, A. F. Murray, J. A. Jackman, P. R. Norton, P. de V. Duplessis and O. Vogt, in L. M. Falicov, W. Hanke and M. B. Maple (eds.), Valence Fluctuations in Solids, North-Holland, Amsterdam, 1981, p. 187.

2 K. Andres, D. Davidov, P. Dernier, F. Hsu, W. A. Reed and G. J. Niewenhuys, Solid State Commun., 28 (1978) 405.

3 Y. Baer, H. R. Ott and K. Andres, Solid State Commun., 36 (1980) 387.

B. Reihl, N. Mårtensson, D. E. Eastman, A. J. Arko and O. Vogt, Phys. Rev. B, 26 (1982) 1842.

A. J. Arko, D. D. Koelling, B. D. Dunlap, A. W. Mitchell, C. Capasso and M. del Giudice, J. Appl. Phys., 63 (1988) 3680.

4 J.-S. Kang, J. W. Allen, M. B. Maple, M. S. Torikachvili, W. P. Ellis, B. B. Pate, Z.-X. Shen, J. J. Yeh and I. Lindau, Phys. Rev. B, 39 (1989) 13529.

5 C. Koenig, $Z$. Phys. B, 50 (1983) 33.

6 C. L. Seaman, M. B. Maple, B. W. Lee, S. Ghamaty, M. S. Torikachvili, J.-S. Kang, L. Z. Liu, J. W. Allen and D. L. Cox, submitted to Phys. Rev. Lett., 67 (1991) 2882.

7 L. Z. Liu, J. W. Allen, C. L. Seaman, M. B. Maple, Y. Dalichaouch, J.-S. Kang, M. S. Torikachvili and M. A. López de la Torre, submitted to Phys. Rev. Lett.

8 D. L. Cox, Phys. Rev. Lett., 59 (1987) 1240.

9 P. Nozieres and A. Blandin, J. Phys. (Paris), 41 (1980) 193.

10 A. M. Tsvelik, J. Phys. C, 18 (1985) 159.

11 P. D. Sacramento and P. Schlottmann, Phys. Lett. A, 142 (1989) 245.

12 I. Affleck and A. W. W. Ludwig, Nucl. Phys. B, 360 (1991) 360.

13 D. L. Cox, submitted to Europhys. Lett.

14 D. L. Cox, submitted to Europhys. Lett.

15 C. M. Varma, P. B. Littlewood, S. Schmitt-Rink, E. Abrahams and A. E. Ruckenstein, Phys. Rev. Lett., 63 (1989) 1996.

16 A. Murasik, J. Leciejewicz and A. Zygmunt, Phys. Status Solidi A, 28 (1975) K107.

17 H. R. Ott, K. Andres and P. H. Schmidt, Physica B, 102 (1980) 148.

18 M. A. Singh, S. L. Smith, S. E. Nagler and W. J. L. Buyers, Solid State Commun, 74 (1990) 439.

19 A. Furrer, A. Murasik and O. Vogt, Helv. Phys. Acta, 50 (1977) 447.

20 K. Winzer, as reported by J. S. Schilling, Phys. Rev. B, 33 (1986) 1667.

21 U. Larsen, J. Appl. Phys., 49 (1978) 1610.

22 P. Fulde, in K. Gschneider and L. Eyring (eds.), Handbook of the Physics and Chemistry of the Rare Earths, North-Holland, Amsterdam, 1978, pp. 295-386.

23 B. Andraka and A. M. Tsvelik, Phys. Rev. Lett., 67 (1991) 2886.

24 W. E. Gardner, J. Penfold, T. F. Smith and I. R. Harris, J. Phys. F, 2 (1972) 133.

25 M. J. Besnus, J. P. Kappler and A. Meyer, J. Phys. F. 13 (1983) 597.

$26 \mathrm{H}$. A. Mook, private communication, 1991.

27 W. Schlabitz, F. Steglich, C. D. Bredl and W. Franz, Physica B, 102 (1980) 321.

28 G. M. Kalvius, S. Zwirner, U. Potzel, J. Moser, W. Potzel, F. J. Litterst, J. Gal, S. Fredo, I. Yaar and J. C. Spirlet, Phys. Rev. Lett., 65 (1990) 2290. 